\title{
Memory for humorous cartoons
}

\author{
STEPHEN R. SCHMIDT and ALAN R. WILLIAMS \\ Middle Tennessee State University, Murfreesboro, Tennessee
}

\begin{abstract}
Incidental memory for three types of cartoons was compared: original cartoons, literal translations of the originals, and weird cartoons created by inserting incongruous material into the literal translations. In Experiment 1, the three types of cartoons were mixed together in lists. In Experiment 2, each list contained only two cartoon types. In both experiments, original cartoons were remembered better than the literal and the weird cartoons, whereas the literal and the weird cartoons were equally well remembered. The detection of incongruities, or attempts to resolve those incongruities, cannot adequately explain the observed humor effects. The results were also inconsistent with both rehearsal and distinctiveness interpretations. Rather, humor per se appears to support good memory performance. Perhaps participants elaborated or gave sustained attention to humorous material at the expense of less humorous material.
\end{abstract}

One of the major goals of memory research is to determine why some events are more memorable than others. This goal can be achieved by studying how variations in the learning materials influence memory. Thus, numerous researchers have investigated the effects of word frequency, word concreteness, imagery value, pleasantness, sentence complexity, sentence bizarreness, and emotional stimuli on memory. However, very little attention has been paid to how humor influences memory. Humor is often employed by educators, advertisers, entertainers, and even politicians to gain our attention and sustain our interest. It is important that we determine how this potent and pervasive stimulus variable influences memory performance.

Previous research has established that humor enhances memory. For example, Kaplan and Pascoe (1977) demonstrated that humorous examples from a lecture were remembered better than serious examples. Similarly, humor can enhance memory for advertisements (Duncan, Nelson, \& Frontzak, 1984; Gelb \& Zinkhan, 1986). However, much of this earlier research provided only anecdotal or quasi-experimental support for the positive effect of humor on memory. When relevant mnemonic variables are controlled, conditions leading to a humor effect are clearly limited. Schmidt (1994) found that humorous sentences were recalled better than nonhumorous sentences in lists containing both sentence types. However, a homogeneous list of humorous sentences was not remembered better than a homogeneous list of nonhumorous sentences. In fact, enhanced recall of humorous sentences was found at the expense of the nonhumorous sentences in the same list. The humor effect was found with intentional but not incidental instructions. Finally, the effects of humor were observed with measures of sentence

We thank the many students who helped us conduct this research. We give special thanks to Constance R. Schmidt for her critical reading of several earlier drafts of this paper. Correspondence should be sent to S. R. Schmidt, Psychology Department, Middle Tennessee State University, Murfreesboro, TN 37132 (sschmidt@mtsu.edu). recall and not with measures of memory for sentence details. These limitations to the impact of humor on memory are of great practical and theoretical interest. The goals of the research presented below were to further delineate the conditions leading to positive effects of humor on memory and to evaluate several explanations for those effects.

One important empirical issue concerning the impact of humor on memory is the potential confound between humor and incongruity. Incongruity results when material is presented out of context, or when unusual combinations of material are presented. Investigations concerning the von Restorff effect, the isolation effect, and the bizarre imagery effect can be viewed as attempts to understand the impacts of incongruity on memory (Schmidt, 1991). Several theorists have argued that incongruity is a source of humor (Deckers \& Devine, 1981; Suls, 1972). In text comprehension, humor may result from violations of readers' predictions concerning upcoming text (Suls, 1972). Readers are "surprised" when their predictions are thwarted, and they search for a rule that allows the ending of the sentence to match the beginning. Consider the following sample sentence from Schmidt (1994): "There are three ways a man can wear his hair: parted, unparted, and departed" (p. 966). The reader does not expect the word departed, which creates an incongruity. The reader then searches for a rule that allows the sentence to make sense. If a rule is found, the incongruity is resolved, and the reader perceives humor. Failure to find an appropriate rule leads to puzzlement with no perception of humor.

Although it is clear that humor often contains incongruities, the role that incongruity plays in determining the effects of humor on memory is unclear. Researchers have argued that incongruity may lead to increased attention to stimuli (Green, 1958; Karis, Fabiani, \& Donchin, 1984; Schmidt, 1991). The putative effects of humor on memory may result from the perception of the inherent incongruities and the resultant increased attention. Alternatively, the attempt to resolve the incongruities contained in humorous material may involve cognitive pro- 
cesses that lead to good memory performance. That is, readers may engage in elaborative processes while searching for a rule to make a humorous sentence comprehensible. This elaboration may lead to increased memory for the humorous material. Finally, the resolution of the incongruity and/or the resultant perception of humor may lead to increased memory for the humorous material. It is important that we disentangle how detecting an incongruity, attempting to resolve that incongruity, resolving the incongruity, and perceiving humor all individually influence memory performance.

A second important issue is determining whether the humor effect is limited to intentional learning conditions. Perhaps the sentences employed in the Schmidt (1994) experiment were simply not funny enough, or perhaps the experiment lacked sufficient statistical power. If the humor effect does occur only in intentional learning, certain explanations of the effect can be easily eliminated. For example, increased attention to incongruity is thought to be an automatic process (Ohman, 1979). If the humor effect is limited to intentional learning, it cannot be the result of an automated attention response to incongruous material.

The fact that the humor effect was limited to intentional learning led Schmidt (1994) to favor a rehearsal explanation of his results. He argued that humorous material received increased rehearsal at the expense of nonhumorous material. Several additional findings supported this conclusion. Recall of humorous sentences had a negative impact on the recall of nonhumorous sentences, suggesting a tradeoff between processing humorous and processing nonhumorous material. Furthermore, the humor effect was limited to within-lists designs. Such designs are necessary before humorous items can be rehearsed at the expense of nonhumorous items.

Despite this early support, recent research has tended to undermine the differential rehearsal hypothesis. VellaBrodrick, Jory, and Whelan (1996) compared memory for humorous and nonhumorous sentences under experimenterpaced and self-paced conditions. Contrary to the differential rehearsal hypothesis, the humor effect occurred in both the experimenter-paced and the self-paced conditions. A rehearsal explanation would be further undermined if the humor effect were to be found in incidental learning.

In summary, the research presented below had two primary motivations. First, we attempted to separate the potential effects of incongruity and humor on memory. Second, we provided further tests for the humor effect under incidental learning conditions. Our research team selected from Gary Larson's published collections cartoons that we judged to be extremely funny. With a stronger manipulation of humor, we hoped to find a humor effect with incidental instructions. Three versions of selected cartoons were created to aid in the separation of humor and incongruity. The first type of cartoon was the original and unaltered version. The originals contained incongruities between the captions and the pictures and were intended to be humorous. In one cartoon, for example, a woman is standing at a window calling her dog.
The dog is pictured running full speed down the front walk toward the front door of the house. The door contains a small dog door that is clearly nailed shut, with the hammer and nails displayed in the foreground of the cartoon. The caption reads: "Here Fifi! C'mon! ... Faster, Fifi!" A second type of cartoon depicted a "literal" translation of the cartoon caption. In this version, we eliminated the humor of the cartoon by removing the incongruent pictorial material. In the example above, all evidence that the door was nailed shut was removed from the picture, including the hammer and nails. A third type of cartoon (weird) was created by adding incongruent information to the literal versions. In the weird cartoons, the incongruity could not be resolved, and consequently these cartoons ought not to have been humorous. In the weird version of our example, the dog was removed from the literal version and replaced by a large snake. The three versions of each cartoon enabled us to contrast the effects of (1) incongruity plus humor (original cartoon), (2) neither incongruity nor humor (literal version), and (3) incongruity with no humor (weird version). Thus, the effects of incongruity and humor on memory could be separated.

\section{EXPERIMENT 1}

In Experiment 1, all three types of cartoons were mixed together in a within-lists design. In addition, incidental learning instructions were employed to determine whether the humor effect could be found under conditions that minimize intentional verbal rehearsal processes.

\section{Method}

Participants. Eighty-four participants from the Psychology participant pool were randomly assigned to three experimental conditions. The participant pool consists primarily of students earning course credit for General Psychology. Students in other psychology courses participated for extra credit.

Materials and Design. Twenty-four cartoons were selected from Larson $(1985,1987,1988)$. Three versions of each cartoon were prepared: original, literal, and weird. The literal versions were created by whiting out incongruous information and adding literal interpretations by cutting and pasting elements from other cartoons. Similarly, the weird versions were creating by pasting incongruent elements from unrelated cartoons into the literal cartoons. All cartoons contained picture elements drawn only by the original artist. In addition, each version of a cartoon contained the same caption. The cartoons were photographed on slide film. The slides were then used to present the material on a screen in front of the students, using a Kodak Ektagraphic slide projector controlled by an electronic timing device. Three experimental conditions were tested that represented the counterbalancing of cartoons across cartoon types. These three arbitrary conditions contained 28, 30, and 26 participants. Each cartoon was presented in its original, literal, and weird forms across participants. Cartoon type was manipulated within subjects.

In a pilot study, 24 participants were asked to use a 5-point scale to rate each slide on four dimensions: familiarity, humor, bizarreness, and comprehensibility. A summary of the rating data is presented in Table 1. Significant differences were observed across cartoon types in the humor ratings $\left[F(2,40)=33.68, M S_{\mathrm{e}}=0.7774\right]$, bizarreness ratings $\left[F(2,40)=4.69, M S_{\mathrm{e}}=1.3082\right]$, and comprehension ratings $\left[F(2,40)=13.55, M S_{\mathrm{e}}=0.7422\right]$. Newman-Keuls 
Table 1

Ratings of the Three Cartoon Types on Four Scales

\begin{tabular}{lcccc}
\hline & \multicolumn{4}{c}{ Rating Scale } \\
\cline { 2 - 5 } Cartoon Version & Humor & Bizarreness & Familiarity & Comprehension \\
\hline Original & 5.10 & 3.92 & 2.67 & 1.56 \\
Literal & 3.08 & 3.21 & 2.55 & 2.61 \\
Weird & 3.25 & 4.27 & 2.22 & 2.87 \\
$F(2,40)=$ & $33.68^{*}$ & $4.69^{*}$ & 0.86 & $13.55^{*}$ \\
\hline$* p<.05$ & & & &
\end{tabular}

$* p<.05$.

tests revealed that the original cartoons were rated as more humorous $(M=5.10)$ than the literal $(M=3.08)$ and weird $(M=3.25)$ cartoons, but the literal and weird cartoons were not significantly different from one another. The humorous and weird cartoons were rated as more bizarre than the literal cartoons, with means equal to $3.92,4.27$, and 3.21, respectively. The difference in bizarreness ratings between the humorous and the weird cartoons was not significant. The comprehension ratings revealed that the humorous cartoons $(M=1.56)$ were easier to understand than the literal $(M=$ 2.61) and weird $(M=2.87)$ cartoons. The difference between the literal and the weird cartoons was not significant.

Procedure. The participants were told that the experiment concerned the relationship between the perception of humor and mathematical abilities. The instructions described the slide presentation of the cartoons and a subsequent arithmetic task. Each slide was presented for $15 \mathrm{sec}$, followed by a 15 -sec rating period. During the rating period, the participants were asked to rate the humor of the cartoons and rate "how difficult is it to get the humor in the cartoon." Following the slide presentation, the students were asked to perform arithmetic for $5 \mathrm{~min}$. They were then allotted $10 \mathrm{~min}$ to recall the cartoons. On the memory test, they were asked to write a brief description of each slide that they remembered and to recall the captions of those slides. The memory test was unexpected.

\section{Results and Discussion}

The probabilities of picture recall, caption recall, and word recall served as measures of cartoon memory. A picture was scored as recalled if the experimenter could uniquely identify the cartoon from a participant's description. Caption recall was scored by a lenient criterion. If the experimenter could identify the cartoon from the recalled caption and at least one word was correctly recalled, the participant was given credit for that cartoon caption. For the probability of word recall, we computed the proportion of words that were accurately recalled; changes in number and tense were allowed, and synonyms were accepted. Two researchers independently scored each of these variables, and discrepancies were resolved. Interrater reliabilities were determined for a subset of the participants $(n=30)$. There were three disagreements in the 720 judgments of picture and caption recall, yielding reliabilities in excess of $99 \%$ for these two measures. Reliability for word recall was $94 \%$. Two additional measures of cartoon memory were derived from the original three. A strict measure of caption recall was defined as the recall of $50 \%$ or more of the words from a caption. We also calculated the probability of word recall, given lenient caption recall (words/caption). This conditional probability provided a measure of memory for detailed caption information. In order to isolate the effects of car- toon type from a potential primacy effect, data from the first three cartoons (one of each type) were excluded from the analyses. The results for each of the memory measures are discussed below and summarized in Table 2. The level of significance for all tests was set at $p<.05$.

A main effect of cartoon type was observed for three of the memory measures: picture recall $\left[F_{1}(2,162)=7.45\right.$, $\left.M S_{\mathrm{e}}=0.2421 ; F_{2}(2,12)=15.35, M S_{\mathrm{e}}=0.1175\right]$, word recall $\left[F_{1}(2,162)=5.09, M S_{\mathrm{e}}=0.0527 ; F_{2}(2,12)=6.00\right.$, $\left.M S_{\mathrm{e}}=0.0447\right]$, and lenient caption recall $\left[F_{1}(2,162)=\right.$ $\left.9.16, M S_{\mathrm{e}}=0.2120, F_{2}(2,12)=13.89, M S_{\mathrm{e}}=0.1398\right]$. With each of these measures, Newman-Keuls tests indicated that original cartoons were remembered better than the literal and weird cartoons. Recall of literal and weird cartoons did not differ significantly. Cartoon type did not significantly affect recall as measured by strict caption recall $\left[F_{1}(2,162)=1.22, M S_{\mathrm{e}}=0.1129 ; F_{2}(2,12)=1.01\right.$, $\left.M S_{\mathrm{e}}=0.1364\right]$ or words recalled per caption recalled $\left[F_{1}(2,100)=0.58, M S_{\mathrm{e}}=0.0106 ; F_{2}\right.$ cannot be calculated when word recall is conditionalized on caption recall].

The different memory measures converge on a common pattern of results. On memory measures sensitive to cartoon access (picture, word, and lenient sentence recall), humorous cartoons were remembered better than literal and weird cartoons. On measures that focus on recall of detailed cartoon information, cartoon type had no effect on memory. These results conform to the pattern reported by Schmidt (1994), who demonstrated that humor affected recall of sentences but not of sentence detail. The results also are consistent with results reported by McDaniel and Einstein (1986), who found that bizarre imagery aided sentence access but not recall of words from accessed sentences.

The present pattern of results helped to distinguish among the effects of detecting an incongruity, processes aimed at resolving the incongruity, and the resolution of the incongruity and perception of humor. The humorous and the weird cartoons were rated equally bizarre; however, the participants remembered more humorous cartoons than weird cartoons. Thus, humor is apparently the potent mnemonic variable with these materials rather than incongruity or bizarreness. Perhaps the humorous cartoons were processed more extensively than the nonhumorous cartoons. The literal cartoons were easy to understand; thus, their processing could have been quickly completed. The humorous cartoons required increased processing as the participants attempted to "get" the car-

Table 2

Probability of Picture Recall, Caption Recall, Word Recall, and Word Recall From Recalled Captions as a Function of Cartoon Type From Experiment 1

\begin{tabular}{lccccc}
\hline $\begin{array}{l}\text { Cartoon } \\
\text { Version }\end{array}$ & $\begin{array}{c}\text { Pictures } \\
\text { Recalled }\end{array}$ & $\begin{array}{c}\text { Words } \\
\text { Recalled }\end{array}$ & $\begin{array}{c}\text { Captions } \\
\text { Lenient }\end{array}$ & $\begin{array}{c}\text { Captions } \\
\text { Strict }\end{array}$ & $\begin{array}{c}\text { Words/ } \\
\text { Caption }\end{array}$ \\
\hline Original & .45 & .16 & .40 & .14 & .38 \\
Literal & .35 & .12 & .29 & .11 & .40 \\
Weird & .37 & .13 & .31 & .12 & .40 \\
\hline
\end{tabular}


toons. However, the participants ought to have processed weird cartoons at least as extensively as the original cartoons, because the weird cartoons were mixed in the humorous cartoons and the participants had every reason to try to "get" the humor in the weird cartoons. The humor effect must be attributed to the actual resolution of the incongruity and/or the resultant perception of humor, rather than to increased processing in search of humor.

Humor clearly enhanced memory under conditions that minimized the influence of intentional verbal rehearsal. The participants did not expect a memory test. Unless there is some intrinsic reason to rehearse humorous material, there was little reason for participants to rehearse in this task. In addition, the complex visual and verbal composition of the cartoons should have made verbal rehearsal difficult. Thus, the results weaken the rehearsal explanation of the humor effect.

Perhaps the humorous cartoons were distinctive within the context of a set containing humorous, weird, and literal cartoons. The distinctive qualities of the humorous cartoons might have led the participants to give those cartoons increased attention or elaboration during encoding. Alternatively, distinctive material might benefit from a special status at retrieval (DeLosh \& McDaniel, 1996), being recalled first and subjected to less output interference than common material. Some support for a distinctiveness interpretation was obtained in an analysis of output order. The output percentiles for original, literal, and weird cartoons were 52.89, 58.25, and 63.24, respectively, revealing a significant main effect $\left[F_{1}(2,122)=\right.$ 4.15]. Additional evidence concerning the role of distinctiveness is presented below.

\section{EXPERIMENT 2}

The purpose of Experiment 2 was to explore the role that list structure plays in producing the humor effect, and to further examine the impacts of incongruity and distinctiveness on memory. In Experiment 1, the lists contained all three types of cartoons, limiting the generality of the results. In Experiment 2, sets of cartoons were created that contained only two types of cartoons. One set contained original and literal cartoons, a second set contained original and weird cartoons, and a third set contained weird and literal cartoons.

Given the results already reported, original cartoons should be remembered better than literal cartoons in the original/literal lists. This result can be explained by any number of hypotheses, including increased attention to incongruous material, increased elaboration in search of humor, distinctiveness, and sustained attention due to humor per se. However, the theoretical options would be narrowed if the humor effect was also obtained in a set composed of half original cartoons and half weird cartoons. According to the incongruity hypothesis, such a set would be equivalent to an all-bizarre list. Original cartoons should not be retained better than the weird cartoons, because both are equally incongruous. This pat- tern of results would also challenge the notion that the humor effect can be attributed to the increased elaboration aimed at resolving incongruities. Participants should attempt to "get" the humor in both the original and the weird cartoons. However, a distinctiveness explanation might still be viable: The original cartoons were both humorous and unusual, whereas the weird cartoons were simply unusual. Humorous material may "stand out" in the context of the nonhumorous weird cartoons, in manner analogous to the effects of bizarreness (McDaniel, Einstein, DeLosh, May, \& Brady, 1995) and orthographic distinctiveness (Hunt \& Elliott, 1980) found in $50 / 50$ lists. The distinctiveness interpretation does not require, a priori, that the humorous cartoons be remembered better than the weird cartoons, but such a finding would not contradict the distinctiveness interpretation. Observation of the humor effect in the original/weird list would be most consistent with the hypothesis that humor, rather than incongruity or distinctiveness, supports good memory for cartoons.

Experiment 2 also provided a way to test the distinctiveness interpretation of the humor effect. Perhaps in Experiment 1 the literal and weird cartoons were perceived as a homogeneous set of "unfunny cartoons." Because the original cartoons were funny, they stood out as a group from these background cartoons. Thus, the original cartoons were remembered best, and no difference was observed between the memory of the literal and that of the weird cartoons. However, if a list contains only weird and literal cartoons, the incongruity in the weird cartoons should make them distinctive and thus they should be recalled better than the literal cartoons. The fact that the weird cartoons were rated as more bizarre than the literal cartoons adds weight to this argument. Alternatively, if the perception of humor produces the memory effects, then literal and weird cartoons should be retained equally well because they were judged as equally humorous.

In summary, four potential explanations of the humor effect were tested in this experiment: incongruity, elaboration in search of humor, distinctiveness, and humor per se. Each explanation makes a different prediction concerning the paired comparisons of the three cartoon types. These predictions are presented in Table 3.

\section{Method}

Participants. One hundred and twenty-eight students were selected from the same source as in Experiment 1.

Materials and Design. The set of cartoons was the same as that employed in the first experiment. The order of the cartoons remained constant across experiments, but the particular versions of the cartoons were rearranged to create the six experimental conditions. These conditions were created by crossing three list structures with two counterbalancing groups of cartoons. Type of cartoon was manipulated within subjects, but each participant viewed only two types of cartoons. One list structure contained original and weird cartoons $(n=44)$; another list structure contained original and literal cartoons $(n=41)$; and a third list structure contained weird and literal cartoons $(n=43)$. Across participants, each cartoon appeared in each form within a given list structure. That is, one group of participants viewed the weird form of Cartoon 1 and the original 
Table 3

Memory Predictions of Four Hypotheses for Each List Structure in Experiment 2

\begin{tabular}{llcc}
\hline & \multicolumn{3}{c}{ List Structure } \\
\cline { 2 - 4 } \multicolumn{1}{c}{ Hypothesis } & Original/ & Original/ & Weird/ \\
Literal & Weird & Literal \\
\hline Incongruity & $\mathrm{O}>\mathrm{L}$ & $\mathrm{O}=\mathrm{W}$ & $\mathrm{W}>\mathrm{L}$ \\
Elaboration in search of humor & $\mathrm{O}>\mathrm{L}$ & $\mathrm{O}=\mathrm{W}$ & $\mathrm{W}>\mathrm{L}$ \\
Distinctiveness & $\mathrm{O}>\mathrm{L}$ & $\mathrm{O} \geq \mathrm{W}$ & $\mathrm{W}>\mathrm{L}$ \\
Humor per se & $\mathrm{O}>\mathrm{L}$ & $\mathrm{O}>\mathrm{W}$ & $\mathrm{W}=\mathrm{L}$ \\
\hline
\end{tabular}

Note-O, Original; L, Literal; W, Weird. See text for an explanation of each hypothesis.

form of Cartoon 2, whereas a second group viewed the original form of Cartoon 1 and the weird form of Cartoon 2.

Procedure. The procedure was nearly identical to that employed in Experiment 1. Participants viewed each cartoon for $15 \mathrm{sec}$, followed by a 15 -sec rating task. They then performed 5 min of arithmetic and were given $10 \mathrm{~min}$ to recall the cartoons. As in Experiment 1 , the memory test was unexpected. The only procedural difference was that participants were asked simply to rate the humor of the cartoons.

\section{Results and Discussion}

In Experiment 1, data from the first three cartoons were discarded prior to the analyses. These three cartoons represented one cartoon of each type. In this experiment, each list contained only two types of cartoons. In order to be as consistent as possible with the previous study, we discarded data from the first two cartoons in Experiment 2. A summary of the memory data is presented in Table 4.

The effects of cartoon type varied as a function of the particular combinations of cartoons contained in the list, with the interactions between list structure and type of cartoon significant for picture recall $\left[F_{1}(2,122)=5.00\right.$, $\left.M S_{\mathrm{e}}=0.1337 ; F_{2}(2,20)=4.46, M S_{\mathrm{e}}=0.2107\right]$, word recall $\left[F_{1}(2,122)=3.61, M S_{\mathrm{e}}=0.0518 ; F_{2}(2,20)=5.21\right.$, $\left.M S_{\mathrm{e}}=0.0359\right]$, and lenient caption recall $\left[F_{1}(2,122)=\right.$ $\left.5.45, M S_{\mathrm{e}}=0.1772, F_{2}(2,20)=6.37, M S_{\mathrm{e}}=0.1516\right]$. This interaction was not significant with strict recall of captions $\left[F(2,122)=1.18, M S_{\mathrm{e}}=0.0940\right]$ or with the conditional probability of word recall given caption recall $\left[F(2,119)=1.03, M S_{\mathrm{e}}=0.0105\right]$.

The LSD tests revealed essentially the same pattern of results when memory for pictures, words, and captions (scored leniently and strictly) was compared across conditions. In each case, original cartoons were recalled better than literal and weird cartoons, and the literal and weird cartoons were retained equally well. A different pattern of results emerged in the analysis of the conditional probability of word recall, given caption recall. Only the difference between the weird $(M=.49)$ and the literal cartoons $(M=.43)$ proved reliable $[F(1,119)=5.98]$. These results replicated those of Experiment 1 and are consistent with the results of Schmidt (1994). In addition, the present results support the position that humor, rather than the incongruity contained in humorous cartoons, leads to good memory. Furthermore, any elabora- tion of humorous cartoons can be attributed to their humor rather than to the fact that they stand out as different from the rest of the cartoons.

In Experiment 1, the memorial consequences of resolving incongruities could not be clearly differentiated from the effects of the perception of humor. The design of Experiment 2 provided an opportunity to separate these effects by assessing recall as a function of rated humor in lists that contained unresolvable incongruities (i.e., the weird cartoons). The recall data were sorted into two groups based on each participant's rating of the individual cartoons to determine whether rated humor alone had a significant effect on recall performance. Cartoons in the upper half of rated humor were recalled significantly better than cartoons in the lower half as measured by picture recall $\left[F(1,122)=10.15, M S_{\mathrm{e}}=\right.$ $0.2337]$, word recall $\left[F(1,122)=8.99, M S_{\mathrm{e}}=0.0670\right]$, lenient caption recall $\left[F(1,122)=11.87, M S_{\mathrm{e}}=0.2232\right]$, and strict caption recall $\left[F(1,122)=5.09, M S_{\mathrm{e}}=0.1504\right]$. None of the interactions with list structure approached significance $(F \mathrm{~s}<1.0)$, suggesting that the most humorous cartoons were remembered better in every combination of cartoon types. We were particularly interested in whether this finding was reliable within the lists containing a combination of literal and weird cartoons. A priori tests revealed that cartoons in the upper half of rated humor were recalled better than those in the lower half even in the literal/weird lists as measured by picture recall $\left[F(1,122)=3.72, M S_{\mathrm{e}}=0.2337, p=.056\right]$, word recall $\left[F(1,122)=4.41, M S_{\mathrm{e}}=0.0670\right]$, lenient caption recall $\left[F(1,122)=4.69, M S_{\mathrm{e}}=0.2232\right]$, and strict caption recall $\left[F(1,122)=4.07, M S_{\mathrm{e}}=0.1504\right]$. These findings further support the claim that humor, rather than bizarreness, distinctiveness, incongruity, or the resolution of incongruities, supports good memory for cartoons.

As in Experiment 1, output percentiles for recalled cartoons were calculated for each cartoon type. There was a marginally significant interaction between cartoon type and list structure $\left[F(2,120)=2.88, M S_{\mathrm{e}}=.0278\right.$, $p=.06]$, indicating that output order was a function of the particular pairing of cartoon types. Newman-Keuls

Table 4

Probability of Picture Recall, Caption Recall, Word Recall, and Words/Caption Recall as a Function of Type of Cartoon and List Structure in Experiment 2

\begin{tabular}{lccccc}
\hline $\begin{array}{l}\text { Cartoon } \\
\text { Version }\end{array}$ & $\begin{array}{c}\text { Pictures } \\
\text { Recalled }\end{array}$ & $\begin{array}{c}\text { Words } \\
\text { Recalled }\end{array}$ & $\begin{array}{c}\text { Captions } \\
\text { Lenient }\end{array}$ & $\begin{array}{c}\text { Captions } \\
\text { Strict }\end{array}$ & $\begin{array}{r}\text { Words/ } \\
\text { Caption }\end{array}$ \\
\hline \multicolumn{7}{c}{ Lists Containing Original and Weird Cartoons } \\
Original & .36 & .18 & .35 & .21 & .52 \\
Weird & .31 & .15 & .30 & .16 & .49 \\
& Lists Containing Original and Literal Cartoons & \\
Original & .45 & .23 & .45 & .25 & .50 \\
Literal & .32 & .16 & .31 & .17 & .49 \\
& Lists Containing Weird and Literal Cartoons & \\
Weird & .35 & .15 & .32 & .18 & .49 \\
Literal & .35 & .14 & .31 & .15 & .43 \\
\hline
\end{tabular}

Note-Cartoon type was manipulated within subjects. 
tests revealed that original cartoons were recalled earlier (mean output percentile $=52.21$ ) than weird cartoons $(M=63.73)$ in the lists containing original and weird cartoons. Original cartoons were also recalled earlier $(M=54.75)$ than literal cartoons $(M=57.24)$ in that list pairing, but this difference was not reliable. No difference in mean output percentile was found in the literal $(M=57.48) /$ weird $(M=57.24)$ pairing.

\section{GENERAL DISCUSSION}

The major findings of this research can be summarized as follows. First, humorous original cartoons were recalled better than literal or weird cartoons. Second, the humor effect occurs under incidental learning instructions, as well as under intentional learning instructions (Schmidt, 1994). Third, cartoon humor led to enhanced recall of cartoon gist, but not increased recall of detailed wording of the cartoon captions. Each of these conclusions, along with its implications, is discussed below.

Researchers have argued that humor arises as a result of incongruity (e.g., Deckers \& Devine, 1981; Suls, 1972). In many studies of bizarre imagery, incongruous sentences have led to better recall than have common sentences (e.g., McDaniel \& Einstein, 1986). Thus, a reasonable hypothesis was that good memory for humorous materials resulted from the incongruities contained in those materials. The cartoons employed in the experiments reported above tested specifically whether incongruity or humor enhanced memory. Both the weird and the original cartoons contained incongruent material, and the weird cartoons were rated as nominally more bizarre than the original cartoons. Nonetheless, the humorous original cartoons were recalled better than the weird cartoons.

In addition, incongruity failed to have an effect on memory even in a contrast between weird cartoons and literal cartoons. Instead, the weird and literal cartoons rated as more humorous in this list structure were recalled better than those rated as less humorous. The incongruities in the weird cartoons could not be resolved easily, suggesting that participants may have perceived humor in these cartoons without fully comprehending them. Furthermore, the literal cartoons did not contain incongruous elements; thus, incongruity resolution could not explain their humorous content either. The memory effects in the reanalysis of the weird/literal condition are therefore more likely due to perception of humor rather than the resolution of incongruities. Of course, some research participants might have found idiosyncratic means of resolving the incongruities in the weird cartoons, and they might have found and resolved incongruities in some of the literal cartoons. These idiosyncratic resolutions could then have been responsible for both the perception of humor and the good recall of the cartoons rated as humorous. It may not be possible to disentangle these two variables. In fact, theories of humor are built on the assumption that the resolution of incongruities causes the perception of humor (e.g., Deckers \& Devine, 1981; Suls,
1972). However, the most direct interpretation of the results of Experiments 1 and 2 is that the perception of humor is more closely related to memory performance than is the putative resolution of cartoons that by definition do not contain incongruities (the literal cartoons) or do contain incongruities that are not easily resolved (the weird cartoons).

The results reported above also provided a test of the distinctiveness interpretation of the humor effect. Two factors initially supported this interpretation: The humor effect appears limited to mixed list designs (Schmidt, 1994), and humorous material is recalled prior to nonhumorous material in the same list. However, the distinctiveness interpretation erroneously predicted better recall of weird cartoons than of literal cartoons. In addition, it does not explain why cartoons rated as humorous were recalled better than those rated as nonhumorous in the weird/literal list.

Schmidt (1994, Experiment 4) failed to find significant differences in the recall of humorous and nonhumorous sentence versions in an incidental memory task. Some of the individual measures of sentence recall (e.g., proportion of words recalled) did reveal reliable effects, but the $F_{2}$ ratios were generally below critical value across the various memory measures. Considering the experiments reported above, we can conclude that the failure to find a significant effect of humor in incidental memory in Schmidt (1994) was a Type II error. Even in the incidental memory conditions reported by Schmidt (1994), rated humor was significantly related to sentence recall. This finding was replicated in Experiment 2, reported above. Clearly, humor enhances memory in both intentional and incidental learning tasks, a finding that requires a modification or extension of the selective rehearsal hypothesis. Differential rehearsal of humorous cartoons was first suggested (Schmidt, 1994) because the humor effect was limited to intentional learning. In addition, simple verbal rehearsal is not possible with the complex visual and verbal nature of the cartoons employed in this series of experiments. In order for the selective rehearsal hypothesis to remain tenable, it must explain why participants rehearsed the humorous cartoons, how that rehearsal took place, and why such rehearsal is not responsive to variations in presentation rate (Vella-Brodrick et al., 1996).

The cartoons employed in the present series of studies allowed us to test memory for captions that remained constant across experimental conditions. On memory measures that reflected access to the gist of the captions (i.e., lenient caption recall), humor enhanced memory. On measures that reflected detailed memory for the captions (the conditional recall of words from captions recalled), no effect of humor on memory was observed. Schmidt (1994) compared memory for humorous sentences written by famous authors such as Mark Twain with memory for nonhumorous sentences written by the experimenters. The sentences created by famous authors were more memorable, a finding that could be attributed to the humor 
effect or to other differences between the sentences. The results reported above were not compromised by such differences in the wordings of the materials. Thus, we can now be more confident that humor does not improve memory for the detailed wording of the humorous material. This finding provides an answer to an interesting puzzle. If humor aids memory, why is it so difficult to retell a joke? Humor helps you remember the gist of the joke, but successfully telling the joke may require memory for its exact wording.

These results provide strong evidence for the mnemonic benefit of humor. In addition, the humor effect was obtained under conditions in which an effect of incongruity or bizarreness was not obtained, suggesting that the humor effect is stronger than the bizarreness effect. Perhaps the many self-help books suggesting the use of bizarre imagery as a memory aid (e.g., Lorayne \& Lucas, 1974) should also include humor as a mnemonic device. In addition, a reevaluation of the many studies demonstrating that bizarreness aids memory may be warranted. Some of the bizarre materials employed in those studies may also have been humorous. Confidence in the bizarreness effect should be weakened until it is demonstrated that the bizarreness effect can be obtained when materials are equated for humor.

Perhaps the best explanation of the humor effect is that humorous material may lead to sustained attention and subsequent elaborative processes. This sustained attention is not simply verbal rehearsal, nor does it require an intention to learn the material. However, Schmidt (1994) found that sustained attention to humorous material is often at the expense of nonhumorous material presented at about the same time. The eventual success or failure of the sustained attention hypothesis will depend on its ability to explain why people pay attention to humorous material at the expense of nonhumorous material.

\section{REFERENCES}

Deckers, L., \& Devine J. (1981). Humor by violating an existing expectancy. Journal of Psychology, 108, 107-110.

DeLosh, E. L., \& McDaniel, M. A. (1996). The role of order information in free recall: Application to the word-frequency effect. Journal of Experimental Psychology: Learning, Memory, \& Cognition, 22, 1136-1146.
Duncan, C. P., Nelson, J. E., \& Frontzak, N. L. (1984). The effect of humor on advertising comprehension. In T. C. Kinnear (Ed.), Advances in consumer research (Vol. 1, pp. 432-437). Valdosta, GA: Association for Consumer Research.

Gelb, B. D., \& Zinkhan, G. M. (1986). Humor and advertising effectiveness after repeated exposures to a radio commercial. Journal of Advertising, 15, 15-20.

GreEn, R. T. (1958). The attention-getting value of structural change. British Journal of Psychology, 49, 311-314.

Hunt, R. R., \& ElliotT, J. M. (1980). The role of nonsemantic information in memory: Orthographic distinctiveness effects on retention. Journal of Experimental Psychology: General, 109, 49-74.

Kaplan, R. M., \& Pascoe, G. C. (1977). Humorous lectures and humorous examples: Some effects upon comprehension and retention. Journal of Educational Psychology, 69, 61-65.

Karis, D., FABIANi, M., \& Donchin, E. (1984). "P300" and memory: Individual differences in the von Restorff effect. Cognitive Psychology, 16, 177-216.

LARSON, G. (1985). Valley of the far side. Kansas City, MO: Universal Press.

LARson, G. (1987). The far side observer. Kansas City, MO: Universal Press.

Larson, G. (1988). Night of the crash-test dummies. Kansas City, MO: Universal Press.

Lorayne, H., \& LuCAs, J. (1974). The memory book. New York: Ballantine.

McDaniel, M. A., \& Einstein, G. O. (1986). Bizarre imagery as an effective memory: The importance of distinctiveness. Journal of Experimental Psychology: Learning, Memory, \& Cognition, 12, 54-65.

McDaniel, M. A., Einstein, G. O., DeLosh, E., May, C., \& Brady, P. (1995). The bizarreness effect: It's not surprising, it's complex. Journal of Experimental Psychology: Learning, Memory, \& Cognition, 21, 422-435.

Ohman, A. (1979). The orienting response, attention, and learning: An information-processing perspective. In H. D. Kimmell, E. H. van Olst, \& J. E. Orlebeke (Eds.), The orienting reflex in humans (pp. 443471). Hillsdale, NJ: Erlbaum.

Schmidt, S. R. (1991). Can we have a distinctive theory of memory? Memory \& Cognition, 19, 523-542.

SCHMIDT, S. R. (1994). Effects of humor on sentence memory. Journal of Experimental Psychology: Learning, Memory, \& Cognition, 20, 953-967.

Suls, J. M. (1972). A two-stage model for the appreciation of jokes and cartoons: An information-processing analysis. In J. H. Goldstein \& P. E. McGhee (Eds.), The psychology of humor (pp. 81-100). New York: Academic Press.

Vella-Brodrick, D. A., Jory, M. K., \& Whelan, T. A. (1996). Effects of humor on sentence recall: A test of the rehearsal and attention hypotheses. Unpublished manuscript.

(Manuscript received November 3, 1997; revision accepted for publication August 15, 2000.) 